\title{
Meiotic outcomes in reciprocal translocation carriers ascertained in 3-day human embryos
}

\author{
Caroline Mackie Ogilvie*,1 and Paul N Scriven ${ }^{1}$ \\ ${ }^{1}$ Guy's and St Thomas' Centre for Preimplantation Genetic Diagnosis, Cytogenetics Department, Guy's, King's and St \\ Thomas' School of Medicine, London and Guy's and St Thomas' Hospital NHS Trust, London, UK
}

Chromosomes involved in reciprocal translocations form quadrivalents at meiosis. These quadrivalents segregate, with or without recombination, to give 32 different meiotic outcomes, only two of which are normal or balanced. This paper presents data collected from 25 cycles of preimplantation genetic diagnosis for 18 couples carrying 15 different reciprocal translocations. Embryos were tested using fluorescence in situ hybridisation with probes for the translocated and centric segments. Overall, $47.7 \%$ (71 out of 149) of embryos tested showed signal patterns consistent with alternate segregation, $24.8 \%$ adjacent- 1 segregation, $10.1 \%$ adjacent- 2 segregation, $15.4 \% 3: 1$ segregation and $2 \% 4: 0$ segregation. For most translocations, alternate segregation was apparently the most frequent mode. Alternate and adjacent- 1 frequencies were similar in male and female carriers; however, 5.7\% of embryos from female translocation carriers showed adjacent- 2 segregation and $20.0 \%$ showed $3: 1$ segregation, whilst the corresponding figures for male carriers were 20.5 and $4.5 \%$. Overall, $2.8 \%$ of embryos were mosaic and $2.3 \%$ of embryos showed chaotic constitutions for the chromosomes tested. The pregnancy success rate for these $\mathbf{2 5}$ cycles was $\mathbf{3 8 . 8} \%$ per embryo transfer and also $\mathbf{3 8 . 8} \%$ per couple.

European Journal of Human Genetics (2002) 10, 801 - 806. doi:10.1038/sj.ejhg.5200895

Keywords: PGD; segregation; reciprocal translocation; meiosis; cleavage stage embryos

\section{Introduction}

Reciprocal translocation is the most common chromosome abnormality, being found in one in 500 people. ${ }^{1}$ Reciprocal translocations have no phenotypic effect in most carriers, but can give rise to reproductive problems, usually recurrent pregnancy loss, chromosomally abnormal offspring, or, in some cases, infertility.

Chromosomes involved in reciprocal translocations form quadrivalents at meiosis. These complexes segregate by alternate, adjacent-1, adjacent-2, 3:1 or 4:0 modes to give gametes with different balanced or unbalanced chromosome complements. ${ }^{2}$ Out of the 32 possible zygotes arising from translocation segregation, only two are genetically balanced, one having normal chromosomes and the other carrying the balanced form of the translocation.

*Correspondence: Dr C Mackie Ogilvie, Cytogenetics Department, 5th Floor, Guy's Tower, St. Thomas Street, London SE1 9RT, UK.

Tel: 0207955 2558; Fax: 0207955 2540; E-mail: caroline.ogilvie@kcl.ac.uk Received 26 February 2002; revised 30 July 2002; accepted 26 August 2002
Empiric data ${ }^{3}$ suggest that only one mode leading to imbalance is likely to result in a viable pregnancy for any one translocation, and Jalbert et $a l^{4,5}$ have published suggested algorithms for determining this 'viable' mode, based on the shape of the pachytene cross formed at meiosis. However, it has been suggested that it is the degree of genetic imbalance in the segregation products which determines the viable mode, rather than the frequency of the products of the different modes in the gametes of the translocation carrier. ${ }^{6}$

The behaviour of these chromosome rearrangements has been studied in male carriers by meiotic analysis in testicular biopsies ${ }^{6-8}$ and by analysis of gametes by fusion of spermatozoa with Chinese hamster oocytes to produce metaphase chromosomes, ${ }^{9,10}$ and using fluorescence in situ hybridisation (FISH) of decondensed sperm heads. ${ }^{11-14}$ These studies have generated some data on segregation mode frequencies in male carriers. However, as noted by Armstrong and Hulten, ${ }^{15}$ sperm chromosome studies cannot differentiate unambiguously between alternate and 
adjacent-1 segregation modes, as chiasmata in the interstitial segments of the translocation chromosomes may produce asymmetric dyads, thereby affecting the spectrum of gametes arising from each mode. These authors found that such chiasmata occurred with a high frequency in the four translocations they had investigated, and pointed out that there is in fact no test currently available for the rigorous discrimination of alternate and adjacent-1 segregation. Thus, where alternate and adjacent-1 segregation products are referred to in this paper, it is the 'balanced' and 'unbalanced' products of these modes that are being discussed.

The lack of direct access to female gametes has meant that until recently data on segregation modes in female carriers had not been easy to collect and had been restricted to studies on foetal ovarian tissue. ${ }^{16}$ With the development of preimplantation genetic diagnosis (PGD) for chromosome rearrangements, these data are now emerging. ${ }^{17-21}$

This paper presents the largest data series yet published on segregation of reciprocal translocations in female and male carriers, ascertained through our PGD programme.

\section{Materials and methods}

Ovarian stimulation, embryo culture, biopsy and spreading were as previously described. ${ }^{20}$

\section{FISH}

Table 1 shows the probe combination used for each translocation. Directly-labelled probes were from Vysis (Vysis Inc., Downers Grove, USA) or QBIOgene (Livingston, UK). Indirectly-labelled probes were also from QBIOgene. Target material and probe were co-denatured at $75^{\circ} \mathrm{C}$ for $5 \mathrm{~min}$, then hybridised for a minimum of $14 \mathrm{~h}$ at $37^{\circ} \mathrm{C}$. Stringent washing to remove unbound probe was in $0.4 \times$ standard saline citrate solution (SSC) at $71^{\circ} \mathrm{C}$ for $5 \mathrm{~min}$. Biotinylated probe was detected with Cy-5-streptavidin (Amersham Pharmacia Biotech UK, Little Chalfont, UK); digoxygenin-labelled probe was detected with FITC- or rhodamine-anti-digoxygenin (Boehringer Mannheim, UK). Preparations were counterstained with DAPI/Vectashield (Vector Labs) and visualised using an Olympus fluorescence microscope, fitted with a 83000 Pinkel filter set and aqua and far red single bandpass filters as required. Images were produced using Quips imaging software (Vysis, UK).

\section{Pachytene shape statistics}

Chromosome segment sizes, excluding heterochromatic and variable regions, were measured using the ISCN ${ }^{22} 850$ band ideogram (in which the relative widths of euchromatic bands are based on direct chromosome measurements). The shape algorithms used were based on those of Jalbert et al, ${ }^{4}$ viz. the ratio of the sum of the centric segments to the sum of the translocated segments and the ratio of the shortest centric segment to the shortest translocated segment.

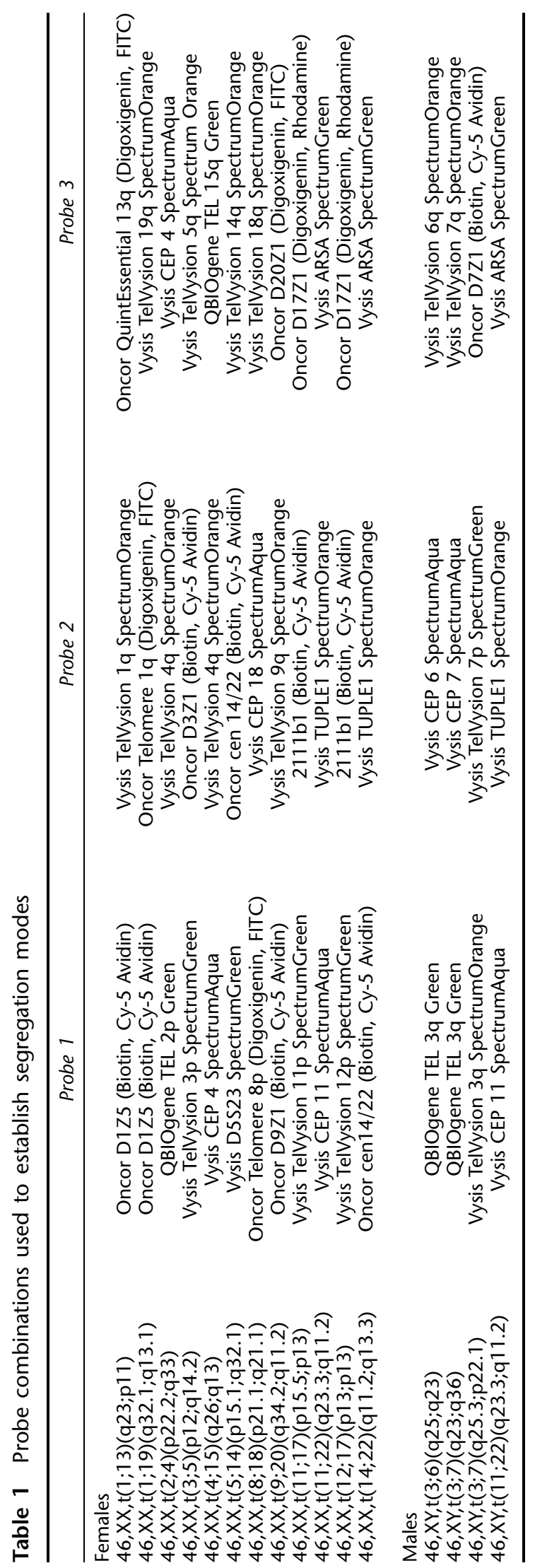




\section{Results}

Twenty-five cycles of PGD were carried out for 18 couples carrying 15 different reciprocal translocations. Eighteen cycles were for female carriers and seven cycles for male carriers. Four cycles were carried out for the common $11 ; 22$ translocation, one for a female carrier, and three for two male carriers. Two apparently unrelated couples had cytogenetically identical translocations between chromosomes 11 and 17; three cycles were carried out. The pregnancy success rate for these 25 cycles was $38.8 \%$ per embryo transfer and $38.8 \%$ per couple.

Pachytene shape algorithms were used to analyse each translocation. The results of this analysis are shown in Table 2 , together with the frequency of the predicted mode in the embryo cohort, expressed both as a proportion of total embryos and as a proportion of embryos with unbalanced products. For the female carriers, three translocations had a shape which predicted $3: 1$ as the likely viable mode; there was a total of 25 embryos from these three translocations, of which seven (28\%) were consistent with $3: 1$ segregation. In this group, there were 12 abnormal embryos, of which $58 \%$ were a result of $3: 1$ segregation. Three translocations showed shapes on the borderline between adjacent- 1 and $3: 1$ predicted modes. $3: 1$ segregation in embryos from this group occurred in two out of 13 (15\%) embryos, and two out of six (33\%) unbalanced embryos. Only one translocation showed a predicted mode of adjacent-2; analysis of embryos showed that 3 out of 10 (30\%) total embryos and three out of four (75\%) unbalanced embryos had arisen following adjacent-2 segregation.
Table 3 shows, for each translocation, the number of embryos in each segregation mode and the percentage overall allocated to each mode. The allocation of embryos to alternate and adjacent- 1 modes assumes either no meiotic crossing-over in the interstitial segments, or an even number of crossover events. Data for male and female carriers are shown separately. Figures 1 and 2 show the distribution of segregation modes for each translocation. In total, 149 embryos were informative; in both male and female carriers, the segregation mode most frequently found (43.2 and $49.5 \%$ respectively) was alternate. Figure 3 shows that the frequency of adjacent- 1 products was also similar (29.5 and 22.9\%) in male and female carriers; frequency of adjacent-2 segregation was higher in male $(20.5 \%)$ than in female carriers $(5.7 \%)$, whilst frequency of $3: 1$ segregation was higher in female $(20.0 \%)$ than in male carriers $(4.5 \%)$. Not all embryos in every cycle were informative for segregation mode, as some embryos had arrested and degenerated at the time of follow-up and did not give informative results. Overall, $2.8 \%$ of embryos were mosaic and $2.3 \%$ of embryos showed chaotic constitutions for the chromosomes tested.

\section{Discussion}

It is known that empirical reproductive risks may be different for male and female carriers of the same translocation. ${ }^{3}$ This may be because quadrivalents behave differently at male meiosis, producing a spectrum of gametes different from that produced at female meiosis. Alternatively, the segregation mode frequency may be similar, but selection may operate against sperm with unbalanced chromosome

Table 2 Analysis of translocations using pachytene shape algorithms to give the predicted segregation mode leading to imbalance

\begin{tabular}{|c|c|c|c|c|c|}
\hline Karyotype & $\Sigma C S / \Sigma T S$ & $\begin{array}{l}\text { Shortest CS/ } \\
\text { shortest TS }\end{array}$ & $\begin{array}{l}\text { Predicted segregation } \\
\text { model leading to imbalance }\end{array}$ & $\begin{array}{l}\text { Predicted mode products } \\
\text { total products }\end{array}$ & $\begin{array}{l}\text { Predicted mode products } \\
\text { total imbalanced products }\end{array}$ \\
\hline \multicolumn{6}{|l|}{ Females } \\
\hline $46, X X, t(2 ; 4)(p 22.2 ; q 33)$ & 6.2 & 8.7 & adjacent-1 & $5 / 7(71 \%)$ & $5 / 5(100 \%)$ \\
\hline $46, X X, t(3 ; 5)\left(p 12 ; q^{14} .2\right)$ & 1.1 & 1.0 & $3: 1$ & $0 / 3(0 \%)$ & $0 / 1(0 \%)$ \\
\hline $46, X X, t(4 ; 15)(q 26 ; q 13)$ & 2.0 & 0.3 & $3: 1$ & $1 / 1(100 \%)$ & $1 / 1(100 \%)$ \\
\hline $46, \mathrm{XX}, \mathrm{t}(9 ; 20)(\mathrm{q} 34.2 ; \mathrm{q} 11.2)$ & 3.6 & 4.2 & adjacent-1/3:1 & $3 / 8(38 \%) / 0 / 8(0 \%)$ & $3 / 3(100 \%) / 0 / 3(0 \%)$ \\
\hline $46, \mathrm{XX}, \mathrm{t}(11 ; 17)(\mathrm{p} 15.5 ; \mathrm{p} 13)$ & 70.0 & 52.0 & adjacent-1 & $7 / 22(32 \%)$ & $7 / 13(54 \%)$ \\
\hline $46, X X, t(11 ; 22)(q 23.3 ; q 11.2)$ & 2.8 & 0.8 & adjacent-1/3:1 & $0 / 3(0 \%) / 2 / 3(67 \%)$ & $0 / 3(0 \%) / 2 / 3(67 \%)$ \\
\hline $46, X X, t(12 ; 17)(\mathrm{p} 13 ; \mathrm{p} 13)$ & 13.4 & 12.8 & adjacent- 1 & $4 / 17(24 \%)$ & $4 / 6(67 \%)$ \\
\hline $46, X X, t(14 ; 22)(q 11.2 ; q 13.3)$ & 0.6 & 1.5 & adjacent-2/3: 1 & $3 / 10(30 \%) / 1 / 10(10 \%)$ & $3 / 4(75 \%) / 1 / 4(25 \%)$ \\
\hline \multicolumn{6}{|l|}{ Males } \\
\hline
\end{tabular}

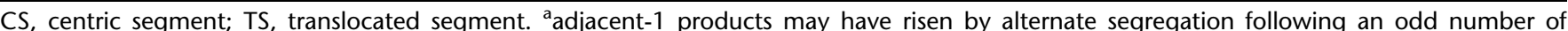
cross-overs in the interstitial segment; adjacent- 2 and 3:1 products can be assumed to represent the acutal meiotic segregation mode; these frequencies are shown in bold. 
Table 3 Number of embryos in each segregation mode for each translocation

\begin{tabular}{|c|c|c|c|c|c|c|}
\hline Karyotype & Alternate* & Adjacent-1* & Adjacent-2 & $3: 1$ & $4: 0$ & cycles \\
\hline \multicolumn{7}{|l|}{ Females } \\
\hline $46, X X, t(1 ; 13)(q 23 ; p 11)$ & 0 & 1 & 0 & 1 & 0 & 1 \\
\hline $46, X X, t(1 ; 19)(q 32.1 ; q 13.1)$ & 11 & 1 & 3 & 6 & 0 & 4 \\
\hline $46, X X, t(2 ; 4)(p 22.2 ; q 33)$ & 2 & 5 & 0 & 0 & 0 & 1 \\
\hline $46, X X, t(3 ; 5)(p 12 ; q 14.2)$ & 2 & 1 & 0 & 0 & 0 & 1 \\
\hline $46, X X, t(4 ; 15)(q 26 ; q 13)$ & 0 & 0 & 0 & 1 & 0 & 1 \\
\hline $46, X X, t(5 ; 14)(p 15.1 ; q 32.1)$ & 4 & 2 & 0 & 3 & 0 & 1 \\
\hline $46, X X, t(8 ; 18)(p 21.1 ; q 21.1)$ & 2 & 0 & 0 & 0 & 0 & 1 \\
\hline $46, X X, t(9 ; 20)(q 34.2 ; q 11.2)$ & 5 & 3 & 0 & 0 & 0 & 1 \\
\hline $46, X X, t(11 ; 17)(p 15.5 ; p 13)$ & 9 & 7 & 0 & 5 & 1 & 3 \\
\hline $46, X X, t(11 ; 22)(q 23.3 ; q 11.2)$ & 0 & 0 & 0 & 2 & 1 & 1 \\
\hline $46, X X, t(12 ; 17)(p 13 ; p 13)$ & 11 & 4 & 0 & 2 & 0 & 2 \\
\hline \multirow[t]{3}{*}{$46, X X, t(14 ; 22)(q 11.2 ; q 13.3)$} & 6 & 0 & 3 & 1 & 0 & 1 \\
\hline & 52 & 24 & 6 & 21 & 2 & 18 \\
\hline & $49.5 \%$ & $22.9 \%$ & $5.7 \%$ & $20.0 \%$ & $1.9 \%$ & \\
\hline Total embryos & 105 & & & & & \\
\hline \multicolumn{7}{|l|}{ Males } \\
\hline $46, X Y, t(3 ; 6)(q 25 ; q 23)$ & 3 & 7 & 2 & 1 & 0 & 1 \\
\hline $46, X Y, t(3 ; 7)(q 23 ; q 36)$ & 3 & 1 & 3 & 0 & 0 & 1 \\
\hline $46, X Y, t(3 ; 7)(q 25.3 ; p 22.1)$ & 4 & 1 & 3 & 1 & 0 & 2 \\
\hline \multirow[t]{3}{*}{$46, X Y, t(11 ; 22)(q 23.3 ; q 11.2)$} & 9 & 4 & 1 & 0 & 1 & 3 \\
\hline & 19 & 13 & 9 & 2 & 1 & 7 \\
\hline & $43.2 \%$ & $29.5 \%$ & $20.5 \%$ & $4.5 \%$ & $2.3 \%$ & \\
\hline \multirow[t]{3}{*}{ Total embryos } & 44 & & & & & \\
\hline & 71 & 37 & 15 & 23 & 3 & 25 \\
\hline & $47.7 \%$ & $24.8 \%$ & $10.1 \%$ & $15.4 \%$ & $2.0 \%$ & \\
\hline Total embryos & 149 & & & & & \\
\hline
\end{tabular}

${ }^{*}$ Allocation of embryos to these modes assumes an even number of, or zero, cross-over events in the interstitial segments (see text).

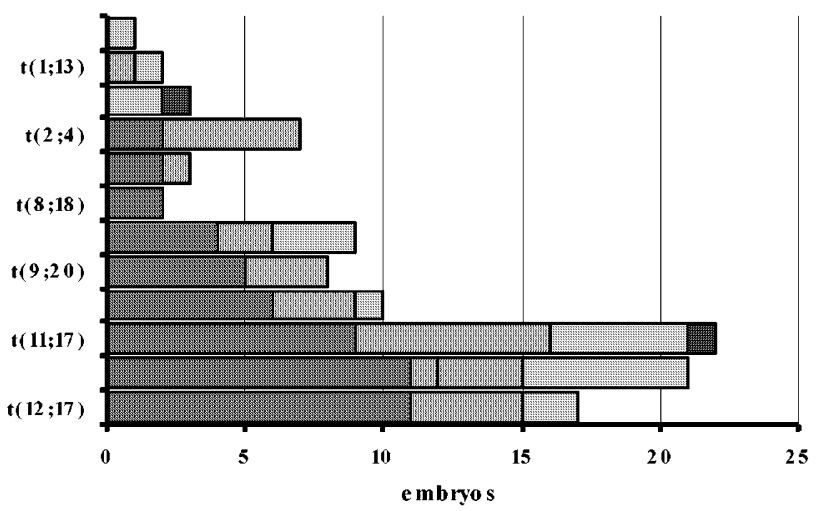

$\square$ alternate $\square$ adjacent $1 \square$ adjacent $2 \square 3: 1 \square$ 4:0

Figure 1 Distribution of segregation modes for 12 different reciprocal translocations in female carriers.

complements, especially if this results in an overall increase in genetic material.

Jalbert et al $^{4}$ published pachytene shape algorithms based on the proportions of the translocated and non-translocated segments and suggested this as a way of predicting for any reciprocal translocation the most likely mode of segregation leading to imbalance. However, studies of 26 different translocations using sperm fusion with Chinese

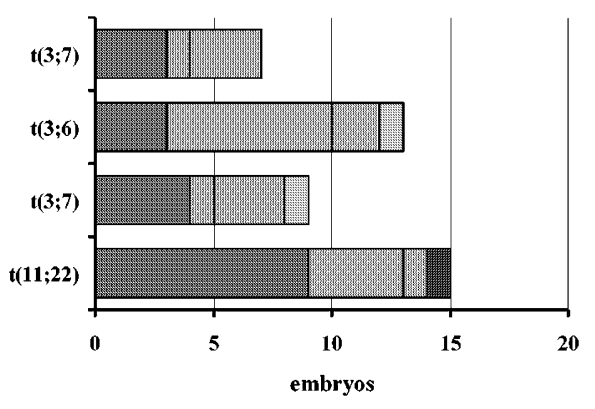

回alternate 回adjacent 1 adjacent $2 \square 3: 1 \square$ 4:0

Figure 2 Distribution of segregation modes for four different reciprocal translocations in male carriers.

hamster ovarian cells (reviewed by Estop et $a l^{9}$ ) showed that in 25 of the 26 translocations, adjacent- 1 was the most frequent mode leading to imbalance, and was found more frequently than alternate segregation in nine translocations. Only one translocation showed a different mode (adjacent-2) as the most common leading to imbalance. This has been followed by papers using FISH to investigate segregation modes in sperm (see above). Alternate segregation was the most frequent mode, and adjacent-1 the most frequent mode leading to imbalance, in sperm of a $t(4 ; 8)$ carrier $^{14}$ and in the sperm of a $t(1 ; 10)$ carrier. $^{12}$ The 


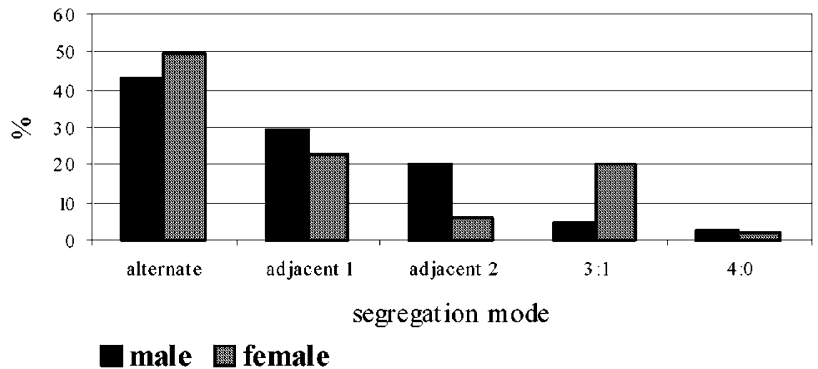

Figure 3 Per cent of total embryos in each segregation mode for male and female carriers.

conclusion from these studies was that the mode predicted by pachytene shape algorithms was likely to reflect selection for the least genetic imbalance and hence to give the most likely viable outcome of any translocation. ${ }^{5}$

We describe the investigation of segregation modes in male and female translocation carriers by FISH on cleavage stage embryos. As the foetal genome is thought not to become active until day $2 / 3$ post-fertilisation, ${ }^{23}$ it is unlikely that any selection would be operating on these early embryos; it can therefore be assumed that, for female carriers, the distribution of segregation modes found reflects the frequency at female meiosis. In the series described here, the overall frequency of alternate and adjacent-1 segregation was similar for male and female carriers, while there was a greater incidence of $3: 1$ segregation in the female carriers than in the male (Figure 3). Although the numbers are still small, the apparent incidence of $3: 1$ segregants in day 3 embryos from male translocation carriers is similar to that found in sperm, ${ }^{9}$ suggesting that there may not be selection against all $3: 1$ products $(3: 1$ monosomy products, for example), at least beyond sperm maturation.

Previous reports of meiotic segregation products in male carriers of the common $t(11 ; 22)$ include studies using sperm fusion with hamster oocytes, ${ }^{24}$ which showed all $2: 2$ and $3: 1$ segregation modes occurring with approximately equal frequency. Estop et al, ${ }^{25}$ using FISH for a carrier of the same translocation, showed only $27 \%$ of spermatozoa as arising from alternate segregation, whereas $40.1 \%$ arose from $3: 1$ segregation. This data would support Jalbert's suggestion ${ }^{4}$ that translocations produce a high frequency of their predicted modes (analysis indicates that 3:1 or adjacent-1 are the predicted mode for this translocation, and empiric data show that $3: 1$ segregation is the only mode that gives rise to viable outcomes). Similar results were obtained by Van Assche et $a l,{ }^{26}$ testing the sperm of the male partner of a couple presenting for PGD for this translocation, but cleavage stage embryos tested did not show a preponderance of $3: 1$ outcomes. These published studies therefore indicate that for this translocation, a preponderance of 3:1 products is seen in sperm, but this preponderance may not be reflected in 3-day embryos. Interestingly, meio- tic studies on testicular tissue from a carrier of the $t(11 ; 22)$ did not find preferential 3:1 segregation. ${ }^{6}$ We carried out three cycles for two male carriers of $\mathrm{t}(11 ; 22)$; in 15 embryos tested, none arose from $3: 1$ segregation.

The results of the pachytene shape analysis for the translocations presented here have been compared with the apparent segregation modes detected 3 days post-fertilisation (see Table 2 and Results section). Interestingly, it would appear that, for the female translocation carriers presented here, pachytene shape analysis may indeed predict the predominant segregation mode leading to imbalance, at least for those translocations where $3: 1$ or adjacent- 2 segregation are predicted. This analysis therefore has value for PGD, as probes schemes can be designed to detect the predicted unbalanced products with appropriate 'internal checks'. ${ }^{2}$ In addition these results may indicate that female meiosis may indeed be different from male meiosis, as direct meiotic analysis of testicular biopsies (ie pre-selection) found no preponderance of the predicted segregation mode over other modes. ${ }^{6}$

Table 3 and Figures 1 and 2 demonstrate that 11 out of the 16 translocations tested showed a preponderance of normal or balanced products. The translocations showing a different pattern may have been due to the poor ovarian response, leading to only a small number of embryos, and hence to sampling error. This preponderance of alternate/ adjacent-1 segregation is consistent with data from sperm studies (see above) and with some published reports on female meiosis ${ }^{17,27}$ and indicates that reports of skewing of segregation modes ${ }^{28}$ may have been the result of cultural artefact or FISH error, especially as it has been shown that a change in embryo culture conditions results in very different outcomes between two cycles for the same couple. ${ }^{20}$

Crossing-over in the interstitial segment has been reported to occur at high frequency in male meiosis. ${ }^{6}$ Whilst it is not possible to detect crossing-over in the interstitial segment followed by alternate or adjacent-1 segregation (see Introduction), an odd number of crossovers in the interstitial segment can be detected when followed by adjacent-2 or 3:1 segregation. ${ }^{2}$ In this series, 27 embryos from female carriers and 11 embryos from male carriers arose following adjacent-2 or 3:1 segregation, and no such recombination event was detected.

In summary, the results presented here show that reciprocal translocations in male and female carriers produce similar frequencies of alternate and adjacent-1 products. However, there is an indication that the frequency of adjacent- 2 and $3: 1$ products may be very different. Comparison of pachytene shape analysis predictions with embryo data indicate that, for female meiosis, translocations may predispose to certain segregation modes depending on the size of the centric and translocated segments. The overall frequency of $47.7 \%$ of genetically balanced embryos in the cohorts available for testing suggests that these reciprocal translocations do not predispose to skewed abnormal 
segregation; this is reflected in the encouraging pregnancy rates for these couples. As more reciprocal translocation carriers present for PGD, further valuable data will emerge on the behaviour of these common chromosome abnormalities at female meiosis.

\section{Acknowledgements}

The authors thank the other members of the Guy's \& St. Thomas Centre for PGD for their support.

\section{References}

1 Hook EB, Hamerton JL: The frequency of chromosome abnormalities detected in consecutive newborn studies - differences between studies - results by sex and by severity of phenotypic involvement.; in Hook EB, Porter LH (eds): Population Cytogenetics. New York: Academic Press, 1977; pp 66-79.

2 Scriven PN, Handyside AH, Mackie Ogilvie C: Chromosome translocations: segregation modes and strategies for preimplantation genetic diagnosis. Prenat Diag 1998; 18: 1437 - 1449.

3 Gardner RJM, Sutherland GR: Chromosome Abnormalities and Genetic Counseling. 2nd edn. Oxford: Oxford University Press, 1996

4 Jalbert P, Sele B, Jalbert H: Reciprocal translocations: a way to predict the mode of imbalanced segregation by pachytenediagram drawing. Hum Genet 1980; 55: 209-222.

5 Jalbert P, Jalbert H, Sele B: Types of imbalances in human reciprocal translocations: risks at birth; in: Daniel A (ed): The Cytogenetics of Mammalian Autosomal Rearrangements. New York: Alan R. Liss, Inc., 1988, pp 267-291.

6 Armstrong SJ, Goldman ASH, Speed RM, Hulten MA: meiotic studies of a human male carrier of the common translocation, $t(11 ; 22)$, suggests postzygotic selection rather than preferential 3:1 M1 segregation as the cause of liveborn offspring with an unbalanced translocation. Am J Hum Genet 2000; 67: 601-609.

7 Goldman ASH, Hulten MA: Meiotic analysis by FISH of a human male $46, \mathrm{XY}, \mathrm{t}(15 ; 20)(\mathrm{q} 11.2 \mathrm{q} 11.2)$ translocation heterozygote: quadrivalent configuration, orientation, and first meiotic segregation. Chromosoma 1993; 102: 102-111.

8 Goldman ASH, Hulten MA: Analysis of chiasma frequency and first meiotic segregation in a human male reciprocal translocation heterozygote, $\mathrm{t}(1 ; 11)(\mathrm{p} 36.3 ; \mathrm{q} 13.1)$, using fluorescence in situ hybridisation. Cytogenet Cell Genet 1993; 63: 16-23.

9 Estop AM, Van Kirk V, Ceiply K: Segregation analysis of four translocations, $t(2 ; 18), t(3 ; 15), t(5 ; 7)$, and $t(10,12)$, by sperm chromosome studies and a review of the literature. Cytogenet Cell Genet 1995; 70: 60-67.

10 Martin RH, Spriggs EL: Sperm chromosome complements in a man heterozygous for a reciprocal translocation $46, \mathrm{XY}, \mathrm{t}(9 ; 13)(\mathrm{q} 21.1 ; \mathrm{q} 21.2)$ and a review of the literature. Clin Genet 1995; 47: $42-46$.

11 Rousseaux S, Chevret E, Moneil M et al: Meiotic segregation in males heterozygote for reciprocal translocation: analysis of sperm nuclei by two and three colour fluorescence in situ hybridization. Cytogenet Cell Genet 1995; 71: 240-246.
12 Van Hummelen P, Manchester D, Lowe X, Wyrobek AJ: Meiotic segregation, recombination, and gamete aneuploidy assessed in a $\mathrm{t}(1 ; 10)(\mathrm{p} 22.1 ; \mathrm{q} 22.3)$ reciprocal translocation carrier by threeand four-probe multicolour FISH in sperm. Am J Hum Genet 1997; 61: 651-659.

13 Estop AM, Cieply KM, Aston CE: The meiotic segregation pattern of a reciprocal translocation $\mathrm{t}(10 ; 12)(\mathrm{q} 26.1 ; \mathrm{p} 13.3)$ by fluorescence in situ hybridization sperm analysis. Eur J Hum Genet 1997; 5: $78-82$.

14 Oliver-Bonet M, Navarro J, Codina-Pascual M, Carrera M, Egozcue $\mathrm{J}$, Benet J: Meiotic segregation analysis in a $\mathrm{t}(4 ; 8)$ carrier: comparison of FISH methods on sperm chromosome metaphases and interphase sperm nuclei. Eur J Hum Genet 2001; 9: 395-403.

15 Armstrong SJ, Hulten MA: Meiotic segregation analysis by FISH investigations in sperm and spermatocytes of translocation heterozygotes. Eur J Hum Genet 1998; 6: 430-431.

16 Hartshorne GM, Barlow AL, Child TJ, Barlow DH, Hulten MA: Immunocytogenetic detection of normal and abnormal oocytes in human fetal ovarian tissue in culture. Hum Reprod 1999; 14: $172-182$.

17 Escudero T, Lee M, Sandalinas M, Munne S: Female gamete segregation in two carriers of translocations involving $2 \mathrm{q}$ and $14 \mathrm{q}$ Prenat Diag 2000; 20: 235-237.

18 Iwarsson E, Malmgren H, Inzunza J et al: Highly abnormal cleavage divisions in preimplantation embryos from translocation carriers. Prenat Diag 2000; 20: 1038-1047.

19 Munne S, Sandalinas M, Escudero T, Fung J, Gianaroli L, Cohen J: Outcome of preimplantation genetic diagnosis of translocations. Fertil Steril 2000; 73: 1209-1218.

20 Scriven PN, O'Mahony F, Bickerstaff H, Yeong C-T, Braude P, Mackie Ogilvie C: Clinical pregnancy following blastomere biopsy and PGD for a reciprocal translocation carrier: analysis of meiotic outcomes and embryo quality in two IVF cycles. Prenat Diag 2000; 20: 587-592.

21 Durban M, Benet J, Boada M et al: PGD in female carriers of balanced Robertsonian and reciprocal translocations by first polar body analysis. Hum Reprod Update 2001; 7: 591-602.

22 ISCN: An international system for human cytogenetics nomenclature; Mitelman F (ed): Basel: S Karger, 1995.

23 Braude PR, Bolton VN, Moore S: Human gene expression firs occurs between the four-and eight-cell stages of preimplantation development. Nature 1988; 333: 459-461.

24 Martin RH: Analysis of human sperm chromosome complements from a male heterozygous for a reciprocal translocation $\mathrm{t}(11 ; 22)(\mathrm{q} 23 ; \mathrm{q} 11)$. Clin Genet 1984; 25: 357-361.

25 Estop AM, Cieply KM, Munne S, Feingold E: Multicolour fluorescence in situ hybridization analysis of the spermatazoa of a male heterozygous for a reciprocal translocation $\mathrm{t}(11 ; 22)(\mathrm{q} 23 ; \mathrm{q} 11)$. Hum Genet 1999; 104: 412 - 417.

26 Van Assche E, Staesson C, Vegetti Wet al: Preimplantation genetic diagnosis and sperm analysis by fluorescence in-situ hybridization for the most common reciprocal translocation $t(11 ; 22)$. Mol Hum Reprod 1999; 5: 682-690.

27 Munne S, Fung J, Cassel MJ, Marquez C, Weier HUG: Preimplantation genetic analysis of translocations: case-specific probes for interphase cell analysis. Hum Genet 1998; 102: 663-674.

28 Conn CM, Cozzi J, Harper JC, Winston RML, Delhanty JDA: Preimplantation genetic diagnosis for couples at high risk of Down syndrome pregnancy due to parental translocation or mosaicism. J Med Genet 1999; 36: 45-50. 